\title{
LA PERTENENCIA SIMULTÁNEA \\ DE LOS ESTADOS EN LOS ACUERDOS \\ DE INTEGRACIÓN EN AMÉRICA LATINA
}

\section{A PARTICIPAÇ̃̃O SIMULTÂNEA DOS ESTADOS NOS ACORDOS DE INTEGRAÇÃO NA AMÉRICA LATINA}

Jorge E. Fernández Reyes

Resumen: En un reciente artículo relacionado con los "Procesos de integración contemporáneos en América Latina", analizábamos en términos generales, las principales tendencias de dichos procesos de integración, individualizando a una de ellas, como la "Superposición e Interrelación" de los mismos en esta región, sin perjuicio de que ésta no era una característica única de América Latina, sino que la misma, también podía observarse en el continente europeo, africano y asiático ${ }^{1}$.

Afirmábamos en dicha oportunidad -sin profundizar en sus consecuencias- que una de las manifestaciones integracionistas de fines del siglo pasado y de lo que transcurre del presente, era la existencia de un número importante de "Procesos de Integración en América Latina", que si bien tenían objetivos o finalidades conceptualmente similares, tenían fundamentos o explicaciones de diversa indole y que se superponían y relacionaban, a partir de la configuración de las "participaciones múltiples" por parte de los Estados.

A partir de dicha afirmación, vinculada con los distintos esquemas de integración en América Latina, la intención de este artículo es analizar el tema desde la óptica de la participación simultánea de los Estados en uno o más Acuerdos Regionales de Integración y las consecuencias que derivan de la misma, del punto de vista económico y jurídico.

Resumo: Em recente artigo relacionado aos "Processos de integração contemporâneos na América Latina", analisamos, em termos gerais, suas principais tendências e, individualmente, a "Superposição e Inter-relação" dos mesmos na região. Tudo sem desconsiderar que não se trata de característica exclusiva da América Latina e que a mesma

\footnotetext{
* Director de la Maestría en Integración y Comercio Internacional de la Facultad de Derecho de la Universidad de Montevideo, Profesor Titular de la Cátedra de Derecho de la Integración de la citada Facultad, ex Director de la Secretaría del MERCOSUR.

1 Los procesos de integración latinoamericanos en la actualidad - Revista de Derecho - Facultad de Derecho - Universidad de Montevideo - Año XI (2012) No. 21, página 31 y siguientes.
} 
também pode ser observada nos continentes europeu, africano e asiático ${ }^{2}$. Afirmávamos, nessa oportunidade e sem nos aprofundar em suas conseqüências, que uma das manifestações integracionistas do final do século passado - eque se dá ainda no presente- tinha lugar na existência de um número importante de "Processos de Integração na América Latina", que, apesar de possuírem objetivos ou finalidades conceitualmente similares, tinham fundamentos ou explicações de indoles diversas, as quais se sobrepunham e relacionavam a partir da configuração das "participações múltiplas" dos Estados.

A partir da referida afirmação vinculada aos distintos esquemas de integração na América Latina, a intenção deste artigo é analisar o tema sob a ótica da participação simultânea dos Estados em um ou mais Acordos Regionais de Integração e as conseqüências que derivam da mesma sob os pontos de vista econômico e jurídico.

Palabras clave: Acuerdos Regionales, Acuerdo de Comercio o Preferenciales de Comercio, Procesos de Integración, Superposición, Interrelación, Pertenencia o Participación Simultánea, Participaciones Múltiples, Cláusula de la Nación Más Favorecida, GATT/OMC (Acuerdo General de Tarifas y Comercio - Organización Mundial de Comercio), Acuerdo Regional, Acuerdo Subregional, Subregionalimo

Palavras-chave: Acordos Regionais, Acordo de Comércio ou Preferências de Comércio, Processos de Integração, Superposição, Inter-relação, Pertencimento ou Participação Simultânea, Participações Múltiplas, Cláusula da Nação Mais Favorecida, GATT/OMC (Acordo Geral de Tarifas e Comércio - Organização Mundial do Comércio), Acordo Regional, Acordo Sub-regional, Sub-regionalismo

\section{Introducción}

A modo de introducción, se puede apreciar en la segunda parte del siglo pasado y en lo que transcurre del presente, una tendencia o realidad imperante en América Latina, en la que participan prácticamente todos los Estados que conforman dicha región, y que responde a la constitución de esquemas de integración de carácter o alcance regional y subregional, de contenido -principalmente- comercial y/o económico, sin perjuicio de contemplar otras dimensiones de la integración (i.e. política, social, cultural, etc.).

\footnotetext{
2 Los procesos de integración latinoamericanos en la actualidad - Revista de Derecho - Facultad de Derecho - Universidad de Montevideo - Año XI (2012) No. 21, página 31 y siguientes.
} 
Dichos Acuerdos Regionales, de contenido comercial y/o económico, encuentran su marco jurídico en la normativa que regula el comercio a nivel mundial (GATT/OMC), y según veremos seguidamente, como una excepción a la aplicación de la cláusula de la nación favorecida (en adelante indistintamente $\mathrm{CN}+\mathrm{F}$ ) de acuerdo a lo dispuesto por el Artículo XXIV del GATT y el Entendimiento sobre la Interpretación del Artículo XXIV del GATT; o la Cláusula de Habilitación; o el Artículo XXV del GATT; o el Artículo V del Acuerdo General del Comercio de Servicios, en función de la categoría de países involucrados y la temática en cuestión, y cuya diferencia radica - esencialmente- en el alcance o profundidad que los Tratados que los constituyen le asignan al proceso de integración en sí mismo, es decir a la modalidad de integración admitida por la normativa vigente y a la que se pretende alcanzar por los Estados integrantes del Acuerdo.

En primer término -y como objeto de análisis de este artículoes posible observar que los Estados participantes de un esquema de integración en particular, ya sea como Estado Miembro, Parte o Asociado o en la categorización que admita el Acuerdo Regional, a su vez se vinculan con otros procesos de integración (sin desvincularse del que podríamos denominar original) en los niveles de participación previstos en esos Acuerdos, surgiendo entonces a partir de estas membresías o participaciones simultáneas de un Estado, la "interrelación" entre los procesos de integración y entre los Estados en el esquema regional, con las consecuencias que ello naturalmente implica en el comercio recíproco, esto es del punto de vista económico y comercial, y en los restantes ámbitos de actuación principalmente en el derecho y los mecanismos de solución de controversias aplicables, aspectos que no han sido objeto de tratamiento intenso por parte de la doctrina especializada.

En segundo término, es dable observar, que los "Procesos de integración" antes referidos cuyo fundamento se inserta en la normativa GATT/OMC (i.e. económico y comercial), incorporan aspectos esencialmente políticos, al igual que otras dimensiones que en algunos casos se vinculan en forma indirecta con los objetivos comerciales o económicos del proceso de integración, pero que en innumerables ocasiones rebasan en forma ostensible, los objetivos iniciales o fundacionales del Acuerdo Regional, los que si bien pueden enriquecer el proceso, también pueden desviar o enlentecer el cumplimiento de la finalidad o los objetivos principales de la modalidad de integración acordada por los Estados signatarios del Acuerdo.

Lo expuesto precedentemente, no inhibe que en ocasiones, las aludidas membresías de los Estados Partes de un Acuerdo Regional 
de integración comercial y/o económica con las características antes expuestas, participe a su vez de esquemas de integración de naturaleza específicamente política o totalmente distinta de la comercial y/o económica, pudiendo generar inconsistencias en los objetivos y acciones a ser desarrolladas en uno y otro u otros esquemas integracionistas.

También, las dificultades interpretativas sobre los aspectos mencionados anteriormente, pueden ocurrir cuando se produce una sucesión en los organismos de integración, dado que en esos casos, se produce la sustitución de un esquema de integración por otro admitiendo modalidades de interacción, al incluir los aspectos orgánicos, institucionales, normativos, etc., en base a un nuevo Acuerdo (Tratado constitutivo) que habitualmente establece renovados objetivos, instrumentos, plazos, etc., aún cuando se inscribe en el marco del Sistema Multilateral de Comercio en el régimen de excepción previsto normativamente.

En resumen, analizaremos, la "participación simultánea" de uno o varios Estados en diversos Acuerdos Regionales de Integración, atendiendo al marco jurídico que regula el "comercio" a nivel mundial aplicable a dichos esquema regionales de integración, así como al tipo de Acuerdo en cuanto a su modalidad de integración, a las dimensiones involucradas en el mismo, y por ende, a la estructura orgánica, y la forma de participación de los Estados conforme a los distintos ordenamientos jurídicos emanados de los Acuerdos constitutivos y sus Protocolos adicionales.

\section{Aspectos previos}

\subsection{Los acuerdos regionales en el marco del GATT y la OMC}

De acuerdo a lo expresado anteriormente, se trata de analizar la "participación simultánea" de los Estados en los distintos "Esquemas de Integración comerciales y/o económicos" (i.e. Acuerdos Regionales, Acuerdos Preferenciales de Comercio, Acuerdos de Libre Comercio, Acuerdos de Preferencias Económicas, etc.), reconocidos como tales por el Acuerdo General sobre Aranceles Aduaneros y Comercio (en adelante indistintamente el GATT) y la Organización Mundial de Comercio (en adelante indistintamente la OMC), y que gozan de la excepción al Principio de No Discriminación, en los términos de la no aplicación de la Cláusula de la Nación Más Favorecida (en adelante indistintamente $\mathrm{CN}+\mathrm{F})^{3}$.

3 La noción de estos Acuerdos Regionales de carácter comercial y/o económico, en términos generales, alude a un Acuerdo entre dos o más Estados que se conceden recíproca 
Al respecto, de acuerdo al marco normativo aplicable a la situación planteada, el eje estructural o rector del GATT y de la OMC, es decir el Libre Comercio y el Sistema Multilateral de Comercio respectivamente, se encuentra en el Principio del Multilateralismo y el Principio de No Discriminación y dentro de éste último, en la aplicación da la Cláusula de la Nación Más Favorecida y la Cláusula del Trato Nacional, sin perjuicio de los restantes Principios que sustentan y rigen el comercio a nivel mundial; esto es: (i) un comercio mas libre de manera gradual, mediante negociaciones que promuevan una reducción de los obstáculos al comercio; (ii) un comercio mas predecible o previsible a través de la consolidación en los límites máximos de los topes arancelarios y la transparencia en las normas públicas; (iii) un comercio más competitivo, mediante la promoción de una competencia leal desalentando las prácticas desleales al comercio; y (iv) la promoción del desarrollo y la reforma económica, en un comercio más ventajoso - en términos de tiempo, flexibilidad y privilegios especiales - para los países en vías de desarrollos o menos adelantados.

Por su parte, la Cláusula de la Nación Más Favorecida, consagra el trato igualitario en materia de comercio para todos los países miembros del GATT/OMC, esto es, si un Estado miembro del GATT/OMC le concede a un país una ventaja, un favor, un privilegio o una inmunidad especial (por ejemplo, la reducción del tipo arancelario aplicable a uno o más productos), dicha ventaja, favor, privilegio o inmunidad, se tiene que extender -inmediata e incondicionalmente- a los restantes países miembros del GATT/OMC.

Y esto se aplica en las tres esferas principales del comercio en que actúa el GATT/OMC, es decir en el comercio de Bienes o Mercancías (Artículo 1 del Acuerdo General sobre Aranceles Aduaneros y ComercioGATT); en el comercio de los Servicios (Artículo 2 del Acuerdo General sobre Comercio de Servicios -en adelante indistintamente AGCS); y en materia de Propiedad Intelectual (Acuerdo sobre Aspectos de los Derechos de Propiedad Intelectual relacionados con el Comercio - en adelante indistintamente ADPIC).

No obstante ello, la normativa GATT/OMC admite diversas excepciones a la aplicación del Principio de No Discriminación en las tres esferas de actuación antes aludidas, es decir en el artículo 3 del GATT en materia de bienes o mercancías; en el artículo 17 del AGCS en materia de servicios; y en el artículo 4 del ADIPC en el ámbito de la propiedad intelectual.

o mutuamente preferencias o concesiones en su intercambio de bienes o mercancías, sin perjuicio de la incorporación de otros aspectos vinculados con el comercio recíproco. 
Específicamente podemos referirnos a las excepciones al Principio de No Discriminación (en las Cláusulas aludidas) de la siguiente forma: (i) a la posibilidad de establecer Acuerdos de Libre Comercio, que se aplique únicamente a los productos objeto de comercio dentro del esquema de integración, sin perjuicio de hacer discriminaciones con relación a los productos de terceros países; (ii) a la posibilidad de establecer un Anexo especial a los productos de los países en vías de desarrollo a sus mercados; (iii) la alternativa de fijar obstáculos al ingreso de productos que se consideren objeto de un comercio desleal procedentes de países específicos; (iv) la invocación de cuestiones ambientes relacionadas con el comercio, siempre que no se adopten con la intención de discriminar arbitrariamente entre los productos provenientes de terceros países y los productos semejantes producidos en el país, o entre productos semejantes importados de otros Estados miembros; (v) la adopción o aplicación de las medidas necesarias para proteger la vida de las personas y de los animales o para preservar los vegetales; y (vi) cuando se adopten medidas relativas a la conservación de los recursos naturales agotables, a condición de que las mismas se apliquen conjuntamente con restricciones a la producción o el comercio nacional.

Nuestra referencia, es a la primera de las situaciones de excepción, vale decir a los "Acuerdos Regionales", que en una primera etapa de la regulación del comercio a nivel mundial, incluía solamente a los bienes o mercancías, y por lo tanto, la conformación de las siguientes modalidades de integración: (i) Áreas de Preferencias Económicas, (ii) Zonas de Libre Comercio; y (iii) Uniones Aduaneras, y posteriormente con la incorporación de otros temas en el marco del Libre Comercio (i.e. servicios, etc.), se incorporan el (iv) Mercado Común y la (v) Unión Económica (y Monetaria) como nuevas modalidades de la integración económica y comercial.

La excepcionalidad de los Acuerdos Regionales de Integración, tuvo su fundamento original en la liberalización del comercio regional, en términos de un avance en la aplicación de las reducciones arancelarias, sin perjuicio de que en la actualidad, la doctrina mayoritaria lo circunscribe a una suerte de laboratorio o etapa previa de preparación a la aplicación de las disciplinas multilaterales.

Independientemente de la fundamentación que se elabore respecto al relacionamento del multilateralismo con el regionalismo, en cuanto a la regla y la excepción, es decir, a la contradicción ínsita de la existencia de los Acuerdos Regionales, en tanto se trata de asociaciones discriminatorias del comercio en la región integrada respecto de terceros 
países, lo cierto es que tanto el GATT y la OMC desde su visión orgánica y normativa los ha considerado compatibles, aplicando la teoría de la complementariedad y de las estrategias sustitutivas, bajo la premisa de que promuevan la "creación de comercio" en la región integrada, y no sean instrumentos de "desvío del comercio".

\subsection{La excepción a la clausula de la Nación más favorecida}

Por lo tanto, y según viene de verse, los "Acuerdos Regionales de Libre Comercio", son una de las excepciones admitidas en el seno del GATT/OMC, a la aplicación de la Cláusula de la Nación Mas Favorecida, en el entendido de que la creación de comercio sea superior a la desviación de comercio.

Esto implica, tanto en el mecanismo estatuido por el Artículo XXIV del GATT y el Entendimiento sobre la Interpretación del Artículo XXIV del GATT (normas y disciplinas que se refieren a las excepciones para los países desarrollados); como en la Cláusula de Habilitación (normas y disciplinas comerciales para los países en vías de desarrollo); en el mecanismo creado para el análisis del Mercado Común del Sur - MERCOSUR (a partir de la consideración de que no era aplicable la Cláusula de Habilitación por ser uno de sus integrantes -la República Federativa del Brasil- un país desarrollado lo que implicó el análisis en una suerte de combinación de ambos mecanismos por parte del Comité sobre Acuerdos Comerciales); y el Artículo V del AGSC (normas para la integración económica en las áreas de servicios); el cumplimiento de determinados requisitos de forma (procedimiento) y de fondo (prueba de compatibilidad, ejercicio de transparencia y aspectos comerciales), que varían - naturalmente -de acuerdo al mecanismo utilizado para obtener el régimen de excepción.

O sea que de acuerdo a lo que viene de observarse, cuando dos o más Estados, suscriben la constitución de un Acuerdo Regional, pueden obtener -a través del mecanismo que corresponda de acuerdo a la condición de los países (desarrollados o en vías de desarrollo) que lo integran y con el cumplimiento de los requisitos establecidos por la normativa vigente para el grado de integración planteada - la excepción de la aplicación de la Cláusula de la Nación Más Favorecida, ante los terceros países miembros del GATT/OMC que no forma parte del Acuerdo Regional.

4 La doctrina especializa la ha considerado para los países en vías de desarrollo, tanto un alternativa como un instrumento de desarrollo económico e inserción a nivel mundial, esto es, una senda o camino o experiencia a una mayor liberalización en las relaciones comerciales internacionales. 
Esto es, obtienen un régimen específico para el comercio recíproco intrazona, es decir entre los Estados Partes (fundadores y que se adhieran), y a su vez, estas ventajas, beneficios, etc. que se otorgan los Estados Miembros o Partes o Asociados, que participan del proceso de integración, no deben ser extendidos a los restantes países miembros de la Organización Mundial de Comercio.

Se trata entonces, a partir del reconocimiento de la condición de Acuerdo Regional en el marco del GATT/OMC, de la existencia en lo "interno del bloque comercial" de un régimen específico aplicable al comercio recíproco entre los Estados que conforman dicho esquema de integración, esto es, la región integrada tiene una regulación derivada del Tratado o Acuerdo constitutivo del esquema de integración, que se aplica, dentro del bloque regional, y que será variable de acuerdo a la modalidad de integración del bloque (i.e. Zona de Libre Comercio, Unión Aduanera, etc.).

A partir de esta consecuencia directa de la aplicación de la excepción de la $\mathrm{CN}+\mathrm{F}$ en lo interno del Acuerdo Regional, si se mantiene la aplicación de la $\mathrm{CN}+\mathrm{F}$ en los términos de la normativa GATT/ $\mathrm{OMC}$ en el comercio recíproco de los Estados integrantes del bloque regional (la multilateralización del comercio como principio básico de las relaciones comerciales entre los países del GATT/OMC), o en su defecto como consecuencia de la excepción referida o por imperio de las características de la modalidad de la integración, el régimen aplicable es el resultante del ordenamiento jurídico del Acuerdo Regional.

Lo que sí es claro, que la excepción a la aplicación de la Cláusula de la Nación Más Favorecida, implica - como venimos de ver - que este régimen de excepción en el comercio recíproco en lo interno (o hacia adentro del bloque regional) no debe extenderse a los restantes países miembros de la OMC, creando la aplicación de la excepción en lo externo del esquema regional, es decir, hacia fuera del mismo, esto es, oponible a terceros países miembros de la Organización Mundial de Comercio.

Parece claro, en una primera lectura, que la excepción de la Cláusula de la Nación Más Favorecida en los Acuerdos Regionales, tiene consecuencias directas en lo interno del esquema regional, y ello genera dificultades interpretativas (y por ende problemas jurídicos), en sede de las adhesiones de los Estados en forma simultánea a dos o más bloques comerciales y/o económicos. 


\subsection{Otros aspectos}

De forma complementaria, y en el párrafo final en el numeral I de este artículo (i.e. Introducción), es dable observar la incorporación de otros aspectos, cuya consideración es necesaria para una correcta evaluación general del tema planteado.

Las diferentes modalidades de integración, admitidas en el marco normativo del GATT y de la OMC, se evalúan en función del grado de profundidad establecido en los propios Acuerdos Preferenciales de Comercio, así como en los instrumentos de política económica y/o comercial que se contemplan en los mismos; lo que trae aparejada una institucionalidad diversa (en lo orgánico y en lo normativo); y consecuentemente la participación (i.e. derechos y obligaciones) de los Estados en dichos Acuerdos no será la misma, si estamos ante una Zona de Libre Comercio, una Unión Aduanera, un Mercado Común o una Unión Económica (y Monetaria).

Y esto no solamente, en la exigencia del cumplimiento de ciertas condiciones específicas que los Acuerdos deben cumplir para obtener el reconocimiento del GATT/OMC, sino en el mecanismo utilizado para obtener dicho reconocimiento a través del Comité de Acuerdos Regionales que recibe y "aprueba" las notificaciones de estos Acuerdos Preferenciales de Comercio.

En ese sentido, el Artículo XXIV del GATT 94 y el Entendimiento sobre la Interpretación del Artículo XXIV del GATT 94, aplicable a los Acuerdos entre países en desarrollo y entre éstos y países en vías de desarrollo para la conformación de zonas de libre comercio y uniones aduaneras principalmente, requieren determinadas exigencias -además dela transparencia- en términos de compatibilidad, que no se encuentran totalmente definidas y que generan dificultades interpretativas (i.e. la noción o el alcance del término "comercio sustantivo", etc.).

Mientras tanto, se puede observar que a través de la Cláusula de Habilitación del año 1979, que habilita los Acuerdos entre países en desarrollo, no se contemplan exigencias sustantivas, salvo en lo que refiere a la transparencia, lo que genera una diferencia relevante entre las modalidades de integración vista anteriormente.

Asimismo, la existencia de un proceso de naturaleza comunitaria o de naturaleza intergubernamental, con las diferencias en cuanto a la existencia de un estructura orgánica institucional comunitaria (que representa los intereses de la comunidad) y un ordenamiento jurídico 
comunitario (cuyas normas supranacionales, de aplicación inmediata y de efecto directo), respecto a órganos de naturaleza intergubernamental (representativos de los intereses de los Estados Partes), y con una normativa de la integración, que debe ser -cuando sea necesarioincorporada a los ordenamientos jurídicos nacionales de los Estados Partes para su vigencia, varían sustantivamente los aspectos planteados en este artículo.

Por último, existen en nuestro continente, varios procesos integración (bajo la modalidad de Acuerdos Preferenciales de Comercio notificados al GATT/OMC y reconocidos como tales) que en sus orígenes, es decir en su Tratado constitutivo, se plantean como objetivo principal la conformación de una modalidad de integración económica y/o comercial, no obstante lo cual, a través del tiempo han incorporado al proceso de negociaciones entre los Estados Partes, aspectos que no fueron objeto de consideración por parte de los negociadores del Tratado fundacional (v.g. aspectos políticos, culturales, educacionales, sociales, etc. $)^{5}$.

También, bueno es señalar, en coordinación con el objeto de este documento, la impronta política a nivel de la creación de organizaciones de carácter regional con un objetivo muy claro de procurar una identidad política regional, que se concrete en un posicionamiento consensuado ante problemas o necesidades comunes a nivel regional ${ }^{6}$.

No obstante, su particularidad o especificidad propia en función del objetivo que da mérito o fundamento a su constitución (i.e. política, social, cultural, etc.), se aprecia claramente, la utilización de los esquemas de integración (de carácter comercial y/o económico) existentes y de los cuales forman parte (total o parcialmente los integrantes de las asociaciones con objetivos esencialmente políticos) con fines de sustento o actuación, lo que implica también dificultades interpretativas en el relacionamiento de los distintos esquemas regionales.

\section{La superposición y la Interrelación}

El fenómeno de la “integración", en tanto, cooperación, colaboración y/o coordinación entre Estados a distintos niveles, y

5 Un claro ejemplo de la afirmación realizada es el MERCOSUR, que en su Tratado fundacional (Tratado de Asunción) sus objetivos eran de contenido netamente comercial y/o económico, no obstante lo cual ha incorporado a lo largo de su evolución, la consideración y regulación de aspectos políticos, sociales, culturales, etc.

6 La UNASUR (Unión de Naciones Suramericanas) y la CELAC (Comunidad de Estados Latinoamericanos y Caribeños), son dos ejemplos de estas manifestaciones integracionistas en América Latina y el Caribe. 
para el caso, en los aspectos comerciales y/o económicos en el marco normativo del GATT/OMC, que se denominan principalmente como Acuerdos Regionales o Acuerdos Preferenciales de Comercio, Acuerdos Comerciales entre otras formas de individualizarlos, y que desde lo genérico se podrían categorizar como "Procesos de Integración" de contenido comercial y/o económico (sin descartar la incorporación de otras "dimensiones" de la integración), contemporáneamente se caracterizaban por distintos aspectos, dentro de los que destacábamos, la "superposición" y la "interrelación" de los esquemas de integración.

La primera característica a destacar es la "superposición" que se va dando con el transcurso del tiempo, en virtud de que los "intentos integracionistas" que - por usar un concepto genérico - han perdido su razón de ser por distintos motivos (cualquiera sea el grado de su evolución o profundidad) no desaparecen fácilmente, es más aún, podríamos decir que en ciertas oportunidades no se disuelven o liquidan totalmente, manteniéndose en forma parcial (en el número de integrantes, en los objetivos, etc.), ya sea para operar una transición a un nuevo Acuerdo, o para subsistir en áreas de interés común, de apoyo o complemento ${ }^{7}$.

Dentro de las alternativas planteadas anteriormente, se encuentra la "sucesión" de los Acuerdos Regionales en el tiempo, ya sea porque no han podido lograr el cumplimiento de su objetivo inicial, o por la necesidad de adecuarse a una nueva realidad contextual (i.e. nuevos objetivos, nuevos instrumentos de política comercial, etc.), donde la misma puede desarrollarse en forma automática mediante la sustitución de un Acuerdo por otro, es decir, mediante la supresión del primero y la creación en forma simultánea del nuevo Acuerdo, o a través de la adhesión gradual de los Estados (total o parcialmente) al nuevo Acuerdo, subsistiendo el anterior hasta que pierda su vigencia por imperio de los hechos.

En principio, cuando la "sucesión" se desarrolla en forma gradual, la transitoriedad implica la subsistencia de ambos Acuerdos y por ende la participación de los Estados en dos esquemas de integración a la vez, sin perjuicio de lo cual, no existirían inconvenientes de índole jurídico, dada la inexistencia de una relación jurídica entre una y otra organización, siendo la adhesión a la nueva "organización" una decisión

7 Un ejemplo de este fenómeno podría ser la SICA (el Sistema de la Integración Centroamericano) que a través de la SIECA (Secretaría de Integración Económica Centroamericana) asiste técnica y administrativamente al proceso de integración económica centroamericano, o el ALBA (la Alianza Bolivariana para los Pueblos de nuestra América) como proyecto de colaboración y complementación política, social y económica entre los países de América Latina y el Caribe. 
voluntaria de los Estados.

Las principales dificultades podrían encontrarse en la negociación del "patrimonio histórico", es decir de todas las concesiones obtenidas u otorgadas mediante los mecanismos previstos en el Tratado que se extingue, ya que su continuación, puede suponer la participación de la totalidad de todos los Estados en el nuevo Acuerdo, o en formal parcial, lo que podría determinar asimetrías en las nuevas relaciones comerciales, al igual que el desmantelamiento de los instrumentos de política comercial existentes o la creación de otros, extremos que necesariamente deberán ser objeto de regulación en las instancias de disolución del Acuerdo y en el Tratado constitutivo del nuevo Acuerdo Regional ${ }^{8}$.

Por su parte, en términos de "interrelación”, dos ejemplos recientes son suficientes para explicar y corroborar esta tendencia, ya que si bien tienen una explicación -a nuestro criterio- principalmente política, no menos cierto es que participan no solamente otros "procesos de integración" como tales, sino también Estados en forma individual, que forman parte de otras modalidades de integración.

El primero de ellos es la "Unión Suramericana de Naciones" (cuya sigla es UNASUR), que se integran por 12 países que a su vez participan activamente en otras instancias de integración a nivel regional y continental, con objetivos similares a los contemplados en las otras instancias regionales ${ }^{9}$.

Los objetivos de la UNASUR se encuentran en el artículo 2 del Tratado constitutivo, quelos enumera de la siguiente forma: "construir, de manera participativa y consensuada, un espacio de integración y unión en lo cultural, social, económico y político entre sus pueblos, otorgando prioridad al diálogo político, las políticas sociales, la educación, la energía, la infraestructura, el financiamiento y el medio ambiente, entre otros, con miras a eliminar la desigualdad socioeconómica, lograr la inclusión social y la participación ciudadana, fortalecer la democracia y reducir las asimetrías en el marco del fortalecimiento de la soberanía e

\footnotetext{
8 Como ejemplos de "sucesión" de procesos de integración en el tiempo, podemos señalar: (i) la ALALC (Asociación Latinoamericana de Libre Comercio) y la ALADI (Asociación Latinoamericana de Integración"; (ii) el CARIFTA (Caribeean Free Trade Association), el CARICOM (La Comunidad Económica del Caribe) y el MCC (Mercado Común Centroamericano; (iii) el Pacto Andino y la CAN (La Comunidad Andina de Naciones.

9 Integran la UNASUR: el Estado Plurinacional de Bolivia; la República de Colombia; la República del Ecuador; la República del Perú; la República Argentina; la República Federativa del Brasil; la República del Paraguay; la República Oriental del Uruguay; la República Bolivariana de Venezuela; la República de Chile; la República Cooperativa de Guyana; la República de Surinam; los Estados Unidos Mexicanos; y la República de Panamá.
} 
independencia de los Estados".

Si cotejáramos la enumeración de estos objetivos, con los plasmados en los distintos procesos de integración -en forma total o parcial- en que participan cualquiera de los países miembros entre sí -bilateral, plurilateral o multilateralmente- podríamos comprobar la "interrelación" mencionada y hasta la "superposición" aludida anteriormente.

El otro de los ejemplos que nos interesa destacar es la "Comunidad de Estados Latinoamericanos y Caribeños” (cuya sigla es CELAC), es una organización de naturaleza intergubernamental, sucesora del Grupo de Río y de las Cumbres de América Latina y el Caribe (CALC), con la finalidad de promover la "Integración y el Desarrollo de los países latinoamericanos", mediante la constitución de un espacio regional propio que una a todos los Estados que forman parte de esta organización.

A nuestro criterio, se trata de una organización con una clara finalidad política y estratégica, es decir una "integración política" fuera de la Organización de los Estados Americanos -OEA, esto es, sin Estados Unidos de Norteamérica- EEUU, ya que se observa la participación de los mismos Estados (salvo EEUU) en dos esquemas políticos con los mismos objetivos, etc.

Si observamos la integración de la CELAC, desde la óptica de los procesos de integración existentes en América Latina y el Caribe, la misma se ordena de la siguiente forma, confirmando lo expresado anteriormente:

a) Acuerdos Regionales- (i) Asociación de Estados del Caribe (AEC); y (ii) Asociación Latinoamericana de Integración (ALADI);

b) Acuerdos Subregionales- (i) Unión de Naciones Suramericanas (UNASUR); (ii) Mercado Común del Sur (MERCOSUR); (iii) Comunidad Andina de Naciones (CAN); (iv) Sistema de Integración Centroamericana (SICA); y la Comunidad del Caribe (CARICOM).

Como viene de verse, no solamente los objetivos de todos y cada uno de los Acuerdos Regionales y los Acuerdos Subregionales, sino también los Estados participantes de cada uno de los Acuerdos y de la propia CELAC, son ilustrativos en cuanto a la mentada interrelación 
entre los distintos fenómenos de integración en América Latina ${ }^{10}$.

\section{La pertenencia o participación simultánea}

Desde una óptica más amplia y a la vez más particular, que la señalada en el numeral anterior (i.e. la "superposición o interrelación de los procesos de integración”), a nuestro criterio, el análisis de la "Pertenencia o Participación Simultánea" de un Estado a dos o más Acuerdos Regionales de integración, es la situación que genera mayores dificultades del punto de vista económico/comercial y jurídico.

Desde el punto de vista teórico, cuando nos referimos a la "Pertenencia o Participación Simultánea" de un Estado en dos o más Acuerdos Regionales de Integración que pueden o no estar vinculados entre sí, estamos aludiendo a un fenómeno de la integración económica y comercial de los tiempos contemporáneos y que ha adquirido especial significación por las repercusiones a nivel del relacionamiento entre los Estados y en el desarrollo de los "Procesos de Integración".

El marco jurídico regulatorio de este fenómeno - a nuestro criteriose encuentra en: (i) la normativa del GATT y de la OMC conforme al desarrollo realizado en los numerales anteriores; (ii) en las disposiciones emanadas de los Tratados constitutivos de los Acuerdos Preferenciales de Comercio y sus Protocolos Adicionales (en función de la matriz constitucional del Estado signatario del Acuerdo en cuestión); y (iii) en la Convención de Viena de 1988 sobre el Derecho de los Tratados entre Estados y Organizaciones Internacionales o entre Organizaciones Internacionales, y la Convención de Viena de 1969 sobre el Derecho de los Tratados.

Dicha "Pertenencia o Participación Simultánea", se manifiesta de diversas formas, que por su variedad, se resuelven de distintas maneras, dentro del marco jurídico antes señalado, y que guardan relación con la superposición e interrelación reseñada en el numeral anterior.

Sin perjuicio de otras variables, que podrán ser objeto de consideración en la categorización que se efectuará, a nuestro criterio,

10 Integran la CELAC: Antigua y Barbuda; República Argentina; Mancomunidad de las Bahamas; Barbados; Belice; Estado Plurinacional de Bolivia; República Federativa de Brasil; República de Chile; República de Colombia; República de Costa Rica; República de cuba; Mancomunidad de Dominica; República del Ecuador; República de El Salvador; Grenada; República de Guatemala; República Cooperativa de Guyana; República de Haití; República de Honduras; Jamaica; Estados Unidos Mexicanos; República de Nicaragua; República de Panamá; República del Paraguay; República del Perú; Santa Lucía; Federación de San Cristóbal y Nieves; San Vicente y las Granadinas; República de Surinam; República de Trinidad y Tobago; República Oriental del Uruguay; y República Bolivariana de Venezuela. 
deben tomarse en cuenta los siguientes aspectos: (i) la modalidad de integración adoptada en el Acuerdo Regional, lo que implica necesariamente la evaluación de los objetivos, los instrumentos de política comercial, la institucionalidad normativa y orgánica, entre otras variables; (ii) las obligaciones resultantes en función del reconocimiento del GATT/OMC al Acuerdo Preferencial de Comercio en cuestión, concedido según el mecanismo utilizado, ya sea entre países en desarrollo, o entre éstos y países en vías de desarrollo, o solamente entre éstos últimos; (iii) el marco jurídico en que se desarrollan estos Acuerdos Regionales; y por último (iv) la categorización de los países participantes del Acuerdo Regional, lo que determina sus derechos y obligaciones (v.g. fundadores, miembros plenos, partes, asociados, observadores, etc.).

Sobre dichas bases, la "pertenencia o participación simultánea" se puede dar de las siguientes formas:

a) La pertenencia o participación simultánea de dos o más Estados a dos o más Acuerdos de Preferencias Económicas, que se encuentran vinculados entre sí, situación que se ha sido tipificada como de "Subregionalismo", es decir requiere la existencia de un Acuerdo Regional y un Acuerdo Subregional, donde el primero de ellos regula y contiene al segundo;

b) La pertenencia simultánea de un Estado a dos o más Áreas Preferenciales de Comercio vigentes y no vinculadas entre sí, por lo que no existe un Tratado o Acuerdo marco o general que regule las diferentes Áreas que tienen un objetivo común en el ámbito de la integración, situación que ha sido categorizada como de "Participaciones Múltiples"; y

c) La pertenencia simultánea de un Estado a dos Áreas Preferenciales de Comercio vigentes, por la desvinculación del Estado de una de ellas, para incorporarse a la otra Área Preferencial, lo que implica que durante la transmisión de una organización a otra, el Estado tendrá una pertenencia simultánea, con un objetivo predeterminado.

En la primera de las situaciones descritas y definida como "Subregionalismo", el presupuesto previo de la misma, es que el Acuerdo Regional, contemple la posibilidad de que se desarrollen en su ámbito interno, un "Acuerdo Subregional", y esto inmediatamente nos lleva a la modalidad de integración adoptada, y la incidencia de la aplicación de la $\mathrm{CN}+\mathrm{F}$ o de la excepción en las relaciones derivadas del Acuerdo Subregional, y de la excepción a la aplicación de la $\mathrm{CN}+\mathrm{F}$ en lo externo, 
hacia los restantes Estados integrantes del Acuerdo Regional y terceros países (miembros del GATT/OMC) que no participan del proceso de integración, en general y cuando se trata de una ventaja, beneficio, etc. que otorga un Estado integrante del Acuerdo Subregional en forma individual.

Por lo tanto, a nuestro criterio, y tratándose de Zonas de Libre Comercio y Uniones Aduaneras, cuando el Acuerdo Subregional (en el marco de un Acuerdo Regional) se realiza entre países desarrollados, sin perjuicio de las obligaciones derivadas del reconocimiento al amparo del Artículo XXIV del GATT y de la respectiva Cláusula de Entendimiento, las preferencias otorgadas entre los Estados Partes de dicho Acuerdo corresponden necesariamente a "lo sustancial del comercio recíproco" (que si bien no se encuentra definido, se estima en un $80 \%$ del comercio recíproco entre los Estados signatarios del Acuerdo), lo que implica, en una primera aproximación al tema, adoptar definiciones en cuanto a la situación de los productos que no se incluyen en "lo sustancial o esencial del comercio recíproco", esto es, se les extiende el régimen de preferencias o no; el régimen de preferencias se realiza en forma progresiva y automática (como en los acuerdos de última generación), o se admiten diferencias de preferencias entre los Estados.

Hechas estas observaciones, y dado que el Acuerdo Regional regula y contiene el Acuerdo Subregional, la compatibilidad adquiere trascendencia, dado que al constituirse éste último, puede requerir la modificación del Acuerdo Regional, a tenor de lo dispuesto en el artículo 41 de la Convención de Viena de 1988, y ello implica la participación de los restantes Estados Partes del Acuerdo Regional no partícipes del Acuerdo Subregional y el contralor por parte del GATT/OMC ${ }^{11}$.

La situación varía cuando se trata del reconocimiento mediante la Cláusula de Habilitación, ya que el mismo, se produce entre países en vías de desarrollo, y por lo tanto, las Zonas de Libre Comercio, y las Uniones Aduaneras, no obligan a las preferencias en lo "esencial" del comercio recíproco, por lo que las preferencias en el ámbito interno, presuponen

11 Dice el artículo 41 - Acuerdos para modificar tratados multilaterales entre algunas de las partes únicamente: 1 . Dos o más partes en un tratado multilateral podrán celebrar un acuerdo que tenga por objeto modificar el tratado únicamente en sus relaciones mutuas: a) Si la posibilidad de tal modificación está prevista por el tratado; o b) Si tal modificación no está prohibida por el tratado, a condición de que: i) No afecte al disfrute de los derechos que a las demás partes correspondan en virtud del tratado ni al cumplimiento de sus obligaciones; ii) No se refiera a ninguna disposición cuya modificación sea incompatible con la consecución efectiva del objeto y del fin del tratado en su conjunto. 2. Salvo que en el caso previsto en el apartado a) del párrafo 1 el tratado disponga otra cosa, las partes interesadas deberán notificar a las demás partes su intención de celebrar el acuerdo y la modificación del tratado que en ese acuerdo se disponga. 
un proceso de negociación, que deberá ajustarse a las condiciones de ambos Acuerdos (i.e. artículo 2 literal c de la Cláusula de Habilitación), pero deberá atenderse necesariamente a evitar discriminaciones arancelarias entre los Estados integrantes del Acuerdo.

Vale decir, y a nuestro criterio, la "concesión de preferencias comerciales" a nivel interno es decir dentro del bloque comercial, con las particularidades antes vistas, se encuentran implícitas en las modalidades de la integración aceptadas por el GATT/OMC, si bien se puede considerar que responden a la aplicación de la $\mathrm{CN}+\mathrm{F}$ en lo interno, y tienen como fundamento la multilateralización del comercio, cuyo respaldo normativo se encuentra en el Principio de No Discriminación.

En suma, y en base al planteo inicial, las preferencias comerciales, en lo interno, cualquiera sea la modalidad de integración adoptada, en principio, debe tender a multilateralizarse por las razonas expuestas, las que forman parte de lo sustancial del comercio recíproco, como aquellas que quedan fueran del mismo, a fin de evitar las discriminaciones entre los Estados partícipes de los Acuerdos Regionales y Subregionales, sin perjuicio de los acuerdos internos que se pudieran realizar por los Estados en términos de excepciones (vía Anexos), adopción en forma gradual, etc., pero el proceso de convergencia hacia un mismo régimen regulatorio debe estar presente, bajo pena de afectar los principios rectores del sistema multilateral de comercio.

Aspecto distinto, es excepción de la aplicación de la $\mathrm{CN}+\mathrm{F}$ en lo "externo", que admite dos ámbitos de análisis, es decir, desde el Acuerdo Subregional con relación a los restantes miembros del Acuerdo Regional; y respecto de terceros países no integrantes del Acuerdo Regional, pero miembros de la OMC, y ello en función de la modalidad de integración y la institucionalidad del proceso.

En la primera de las situaciones, parece claro, que no se trata de exceptuarse de la aplicación de la $\mathrm{CN}+\mathrm{F}$, salvo disposición expresa, ya que el Acuerdo Subregional estaría condicionado a que estuviera abierto a la adhesión de los restantes integrantes del Acuerdo Regional, dado que el objetivo ínsito en estos mecanismos de integración es procurar la convergencia hacia la multilateralización del comercio ${ }^{12}$.

12 Un ejemplo de esta situación, se encuentra en la ALADI, con la instrumentación de Acuerdos Subregionales a través de los Acuerdos de Alcance Parcial (AAP) de Complementación Económica (ACE) que contienen - a título ilustrativo - un Programa de Liberación Comercial que establece desgravaciones arancelarias, lineales, progresivas y automáticas, en las que no participan todos los Estados integrantes del Acuerdo Regional, pero necesariamente deben estar abiertos a la adhesión de los restantes Estados, previa negociación, propiciando la convergencia a nivel regional. 
Aspecto distinto, sería la segunda situación, ya que en la misma, es plenamente aplicable la excepción de la $\mathrm{CN}+\mathrm{F}$ por parte de los Estados integrantes del Acuerdo Subregional, según surge de la normativa aplicable de acuerdo a los mecanismos de reconocimiento aplicables, en cualquiera de las modalidades de integración.

La distinción se podría encontrar, en que dicha aplicación también resulta de la existencia de una "política comercial común" que deben adoptar los integrantes del Acuerdo Subregional, cuyas decisiones variarían si las mismas emanasen de organismos intergubernamentales o supranacionales, y tendrá carácter preceptivo o no de acuerdo a la modalidad adoptada o en que se encuentra el proceso de integración.

La situación más compleja, en estos casos, estaría dada por la concesión de una "preferencia" por parte de un Estado que forma parte tanto del Acuerdo Regional, como del Acuerdo Subregional a un tercer Estado ajeno a estos Acuerdos pero miembro del GATT/OMC, y que éstas se realicen desde el Acuerdo Subregional, lo que a nuestro criterio, determina que la preferencia concedida se multilateraliza, por aplicación de la normativa general.

La segunda de las situaciones, la definimos como "participaciones múltiples", y éstas se basan - en una primera modalidad -en la existencia de un Estado que pertenece simultáneamente a dos o más "Acuerdos Preferenciales de Comercio", que a diferencia de la situación anterior, no se encuentran vinculados entre sí.

Esta modalidad si bien -en principio- es posible del punto de vista jurídico, dependerá en primera instancia, de las disposiciones que regulan uno y otro Acuerdo, en función de que admitan la existencia de Estados que participen simultáneamente de dos Áreas Preferenciales de Comercio, con el mismo o diferente "status" en su condición de integrante de los Acuerdos ${ }^{13}$.

El planteo en estos casos, se encuentra, en sí las preferencias concedidas en el marco de uno u otro Acuerdo Regional, se deben extender a los otros Estados integrantes de Acuerdo, es decir, si corresponde la aplicación de la $\mathrm{CN}+\mathrm{F}$ en la relación entre ese Estado que participa de los dos o más Acuerdos Regionales.

La solución a este aspecto, rebasa el alcance de este artículo, dado

13 Un ejemplo de esta modalidad, sería la situación de la República de México con su participación como Estado miembro de la ALADI y a su vez como integrante del NAFTA (Zona de Libre Comercio de Norteamérica). 
que correspondería ingresar al tipo de modalidad de la integración en cuestión, el nivel de participación del Estado en cada uno de los Acuerdos Regionales, y el tipo de preferencia a que nos estaríamos refiriendo, sin perjuicio de lo cual, a nuestro criterio, y siguiendo los lineamientos desarrollados con anterioridad, si no existiese un "acuerdo interno" a nivel del o de los Acuerdos, corresponde la "multilateralización" de la preferencias, es decir la aplicación de la $\mathrm{CN}+\mathrm{F}$ a lo interno de los esquemas regionales de integración ${ }^{14}$.

Por último, y como una segunda modalidad de las participaciones múltiples, nos encontramos ante situaciones en las cuales, un Estado partícipe de un Acuerdo Regional o Subregional, pretenda realizar otro Acuerdo Preferencial con un tercer Estado en forma bilateral, desde el Acuerdo, es decir sin abandonar el Acuerdo Regional o Subregional participar en una relación económica/comercial bilateral con un tercer Estado (sea este partícipe o no de otro Acuerdo Preferencial de Comercio).

Dos son las consideraciones que tradicionalmente se han utilizado a este respecto.

La primera de ellas de contenido estrictamente jurídico, es estar a las disposiciones emanadas del Tratado constitutivo del Acuerdo Regional o Subregional o las disposiciones complementarias adoptadas conforme al ordenamiento jurídico propio, en cuanto a las posibilidades o limitaciones que tienen los Estados integrantes de dichos Acuerdos, para desarrollar negociaciones y concluir Acuerdos bilaterales con terceros Estados ${ }^{15}$.

La segunda de ellas, a nuestro criterio, carente de toda procedencia, recoge los lineamientos de las modalidades de la integración al amparo del GATT/OMC, es decir, si por la instancia o etapa en que se encuentra del proceso de integración o por la modalidad adoptada, corresponde la aplicación de una "política comercial común", las negociaciones y

14 En esencia, no es lo mismo, la condición de Estado Asociado en el MERCOSUR de Chile, Bolivia, etc. en base a la suscripción de Acuerdos de Alcance Parcial con un Programa de Liberación Comercial entre las Partes, que la figura de país Observador de Uruguay o Paraguay en la Alianza del Pacífico, aspectos que podrán cambiar sustantivamente si los Estados Asociados del MERCOSUR ingresan como Miembros plenos, al igual que Uruguay o Paraguay de la Alianza del Pacífico.

15 Un caso que puede ejemplificar el planteo se encuentra en la posición de la República Oriental del Uruguay, en tanto Estado integrante del MERCOSUR, que inicia negociaciones con los Estados Unidos de Norteamérica, con la finalidad de suscribir un Tratado de Libre Comercio (TLC), lo que generó la oposición diplomática de la República Federativa del Brasil, en base al Tratado de Asunción y la Decisión del Consejo del Mercado Común No. 32/00, que establecen que la política comercial se hará en común por el MERCOSUR en tanto organización internacional, y no por los Estados Partes a título individual. 
eventualmente Acuerdos de Comercio, requieren la participación de la Organización, a través de su representación vinculante conforme al Tratado constitutivo, sus protocolos adicionales o la normativa emanada de los órganos del Acuerdo. ${ }^{16}$

La última de las hipótesis planteadas, trata la desvinculación de un Estado integrante de un Acuerdo de Comercio, para incorporarse a otro Acuerdo de Comercio, lo que implica que en la etapa de transición, entre la desvinculación y la incorporación se pertenezca a dos Acuerdos Preferenciales en forma simultánea, que si bien no es estrictamente una "sucesión", reconoce de alguna forma el mismo tratamiento a dicha modalidad de participación simultánea ${ }^{17}$.

\section{Conclusiones}

La "pertenencia o participación simultánea" en las modalidades y términos vistos precedentemente, y conforme al desarrollo realizado puede traer aparejado dificultades en la regulación de las relaciones comerciales entre los Estados entre sí en el ámbito de los Acuerdos Regionales en cuestión y entre los propios Procesos de Integración.

Asimismo, consideramos que los mecanismos de solución de controversias aplicables en las relaciones entre los Estados en el régimen aplicable en el marco de los Acuerdos, también ingresa al ámbito de las dificultades de aplicación en la práctica, admitiendo interpretaciones diversas, aún cuando existan previsiones expresas al respecto en los Protocolos que comprenden las alternativas dadas para la solución de los diferendos entre las Partes (sin perjuicio de otros ámbitos de actuación).

En sustancia, y sin perjuicio de que cada Acuerdo, contempla habitualmente, distintos mecanismos de solución de las controversias que se suscitan entre los Estados integrantes del Acuerdo, ya sea en forma elemental, mediante negociaciones diplomáticas o directas entre los negociadores, con soluciones a nivel de los órganos de administración del Acuerdo, o a través de instancias jurisdiccionales, bueno es señalar, que todos estos Acuerdos Regionales se encuentran en el marco del

16 Este argumento también fue utilizado - aunque con menor intensidad - en la situación descrita en la cita anterior.

17 Esta situación reconoce variados antecedentes en los procesos de integración en América Latina, y recientemente ha tenido aplicación práctica, con la decisión adoptada por la República Bolivariana de Venezuela, al desvincularse de la Comunidad Andina de Nacionales y solicitar su ingreso como Estado Parte del MERCOSUR, donde adquirió previamente la condición de Estado Asociado (a través de una ACE en el ámbito de la ALADI) y luego a través del Protocolo de Adhesión suscrito con el MERCOSUR comenzaron las instancias de la transición para su ingreso. 
GATT/OMC, en tanto miembros de dicha Organización Internacional y por la tanto sometidos a sus reglas, y naturalmente al régimen de solución de diferencias establecido en sus documentos constitutivos y protocolos adicionales.

Como consecuencia de ello, los Estados involucrados en dos o más Acuerdos Regionales, en principio pueden acudir a cualquiera de las instancias previstas para la solución de los diferendos, ya sea a nivel de la OMC, ya sea a nivel del mecanismo del Acuerdo (y en su caso a los mecanismos previstos en los Acuerdos Subregionales), o de los dos (o de los tres), pudiendo generarse "resoluciones" y hasta "laudos arbitrales" dispares en cuanto a un mismo objeto controvertido, siendo la jurisprudencia resultante de la actuación de los órganos jurisdiccionales otro elemento que pueden generar diversidad en las resoluciones conforme a la sede o foro en que se plantea la controversia, al igual que el derecho aplicable por los órganos o tribunales actuantes en cada caso.

La soluciones podrían resultar de: (i) la "jurisdicción obligatoria” de los procedimientos o mecanismos establecidos en el Acuerdo Regional o en el Subregional, lo que a nuestro juicio, colide abiertamente con la génesis de los mismos y por ende la aplicación del régimen estatuido en el GATT/OMC, o en su defecto a través (ii) de la "opción de foro", y si bien significa una avance al respecto, no soluciona la cuestión de fondo.

Visto desde la óptica de la normativa aplicable, y sin perjuicio de la regulación resultante de los Acuerdos a nivel de GATT y de la OMC, necesariamente incorporados a los ordenamientos jurídicos nacionales de los Estados miembros de la Organización, no menos cierto es que, de las fuentes del derecho y el derecho aplicable, puede resultar asimetrías que demuestran la falta de regulación de las situaciones de pertenencia o participación múltiple en los términos vistos anteriormente.

La importancia cuantitativa (en su número y países que participan) y cualitativa (dado que incorporan tanto mercancías o bienes como servicios) de los Acuerdos Comerciales preferenciales que en los últimos años han sido notificados a la $\mathrm{OMC}$, donde predominan significativamente los Acuerdos de Libre Comercio y en una menor medida las Uniones Aduaneras, son ilustrativos en cuanto a las modalidades negociales que utilizan los países y naturalmente inciden en el Sistema Multilateral de Comercio regulado por la OMC.

A su vez, el fenómeno de la vecindad geográfica (i.e. límites fronterizos) no es determinante en el relacionamiento comercial de los 
países, adquiriendo relevancia el comercio regional, sin perjuicio de las negociaciones a nivel global, con preferencia a la bilateralidad.

Aunado a estas consideraciones, se encuentra el objeto de este artículo, es decir, la participación o pertenencia simultánea de los países o dos o más Acuerdos de Comercio.

Todos estos elementos, demuestran la variabilidad de las relaciones comerciales y/o económicas entre los países integrantes de la Organización Mundial de Comercio, y como consecuencia de ello, la dificultad de la regulación de las mismas, lo que adelanta la necesidad de una rápida adecuación de las reglas del comercio a esta nueva realidad, derivada tanto de la proliferación de Acuerdos Preferenciales de Comercio, y corolario de ello, también la pertenencia o participación simultánea de los países en dos o más Acuerdos de Comercio.

Lo básico radica en determinar la incidencia de la aplicación de la $\mathrm{CN}+\mathrm{F}$ en lo interno de los Acuerdos, y la excepción de la aplicación de la $\mathrm{CN}+\mathrm{F}$ en lo interno y en lo externo, definiendo la multilateralización o no de las preferencias concedidas, en las situaciones objeto de consideración en este artículo.

A partir de allí, las diferencias del tratamiento de los instrumentos de política comercial (programas de liberación comercial, períodos de desgravación, medidas y restricciones no arancelarias, excepciones, régimen de origen, cláusulas de salvaguardias, regimenes especiales de importación, zonas francas, admisión temporaria, obstáculos técnicos al comercio, "dumping", liberalización del comercio de servicios, etc.) de cada Acuerdo, deben ser objeto de reglas claras, transparentes y comprensivas de esta nueva realidad que - sin lugar a dudas - está incidiendo en el comercio mundial, y en ello la Organización Mundial de Comercio tiene un gran desafío por delante. 